\title{
Elaboración de un proceso tecnológico para la producción de jugo de naranja en polvo
}

\section{Elaboration of a technological process to produce orange juice powder}

\author{
Alexandra Isabel Tapia Borja. ${ }^{1}$, Mabel Mariela Parada Rivera. ${ }^{2}$, César Arturo Puente \\ Guijarro. ${ }^{3}$, Paúl Marcelo Manobanda Pinto. ${ }^{4}$ \& Gonzalo Iván Guanoluisa Ataballo. ${ }^{5}$
}

\begin{abstract}
.
DOI: https://doi.org/10.33262/cienciadigital.v4i3.1323

El The objective of this work was to evaluate a technological process for the production of orange powder, identifying variables and parameters through tests at the laboratory level, which will later be simulated in a process software in order to analyze its technical and economic feasibility. Two varieties of orange were characterized by analyzing their physicochemical properties, selecting the Valencia variety with $79.78 \%$ and discarding the Washington variety with $61.5 \%$, clarifying and encapsulating the fluid for atomization. Statistically, an excellent yield of $76.7 \%$ and a humidity of $0.763 \%$ were determined, with a temperature of $140{ }^{\circ} \mathrm{C}$ and a concentration of $7 \% \mathrm{w} / \mathrm{w}$. The physical chemical and microbiological analyzes are: ${ }^{\circ}$ Brix; 9.6, $\mathrm{pH}$; 3.61, total coliforms, faecal coliforms, Ecoli $0 \mathrm{CFU} / \mathrm{g}$, molds and yeasts < $10 \mathrm{CFU} /$ $\mathrm{g}$, being within the allowed range of the NTE INEN 2471. The simulation was performed based on 5 (MT) that corresponds to $25 \%$ of the unused production in the orange production of the Caluma canton, taking into account that the total production is $22,482 \mathrm{MT}$, three sections were defined: fruit preparation, juice production and powder production, the same that count with unit operations of each section. From a technical point of view, the project is totally viable, because the sub-processes are feasible to be implemented and the final product meets the necessary requirements for transportation and consumption.

The process is economically feasible, however the system in 15 years generates a NPV (Net Present Value) of 744,000 USD and an IRR (Internal Rate of Return) of 10\% which, despite

\footnotetext{
1 Docente Investigador, Facultad de Ciencias Agropecuarias y Recursos Naturales, Carrera de Agronomía, Universidad Técnica de Cotopaxi (UTC), Latacunga, Ecuador, alexandra.tapia@utc.edu.ec

${ }^{2}$ Docente Investigador, Escuela Superior Politécnica de Chimborazo (ESPOCH), Facultad de Ciencias, Escuela de Ingeniería Química, Riobamba, Ecuador, mparada@espoch.edu.ec

${ }^{3}$ Docente Investigador, Escuela Superior Politécnica de Chimborazo (ESPOCH), Facultad de Ciencias, Escuela de Ingeniería Química, Riobamba, Ecuador, cesar.puente@espoch.edu.ec

${ }^{4}$ Docente Investigador, Universidad Estatal Amazónica (UEA), Departamento de Ciencias de la Vida, Carrera Ingeniería Ambiental, Puyo, Ecuador, pmanobanda@uea.edu.ec

${ }^{5}$ Investigador Independiente en el área ambiental, Latacunga, Ecuador, gonza.guanoluisa01@ @otmail.com
} 
being positive, may not be attractive to investors, considering that a total capital investment ( $\$$ $2,878,000$ \$ USD) and the operating cost $(4,814,762$ \$ USD) will be required.

Keywords: chemical engineering and technology, maltodextrin, spray drying, computational simulation.

\section{Resumen.}

El objetivo de este trabajo fue evaluar un proceso tecnológico para la producción de polvo de naranja identificando variables y parámetros a través de ensayos a nivel laboratorio, que posteriormente se simulo en un software de procesos con el fin de analizar su pre factibilidad técnica y económica. Se caracterizó dos variedades de naranja analizando sus propiedades fisicoquímicas, seleccionando la variedad valencia con un $79.78 \%$ y descartando la Variedad Washington con el $61.5 \%$, se clarifico y encapsulo el fluido para su atomización. Estadísticamente se determinó un excelente rendimiento de $76.7 \%$, y una humedad $0.763 \%$, con temperatura de $140^{\circ} \mathrm{C}$ y concentración $7 \% \mathrm{p} / \mathrm{p}$. Los análisis fisicoquímicos y microbiológico son: ${ }^{\circ}$ Brix; 9.6, $\mathrm{pH} ; 3.61$, coliformes totales, coliformes fecales, Ecoli 0 $\mathrm{UFC} / \mathrm{g}$, mohos y levaduras $<10 \mathrm{UPC} / \mathrm{g}$, encontrándose dentro del rango permisible de la NTE INEN 2471. La simulación se realizó en función a 5 (TM) que corresponde al $25 \%$ de la producción no utilizada en la comercialización de naranja del cantón Caluma, teniendo en cuenta que la producción total es de $22.482 \mathrm{TM}$, se definieron tres secciones: preparación de la fruta, producción de jugo y la producción de polvo, los mismos que, cuentan con operaciones unitarias propias de cada sección. Desde el punto de vista técnico, el proyecto es totalmente viable, debido a que los subprocesos son factibles de ser implementados y el producto final cumple con los requisitos necesarios para su comercialización y consumo. El proceso es económicamente factible, sin embargo, el sistema en 15 años genera un VAN (Valor Actual Neto) de 744.000USD y un TIR (Tasa Interna de Retorno) de 10\% que a pesar de ser positivos podrían no ser atractivos para los inversores, considerando que se requerirá una inversión total del capital (\$2.878.000\$USD) y el costo de operación (4.814.762\$USD).

Palabras Clave: Ingeniería y Tecnología Química, Maltodextrina, Secado Por Atomización, Simulación Computacional.

\section{Introducción.}

La naranja es nativa de la región tropical y subtropical de Asia, desde donde se ha dispersado su cultivo por diferentes partes del mundo y que en la actualidad se produce en varias regiones que poseen un clima cálido y templado. El jugo de naranja al ser consumido en estado fresco genera una fuente natural de nutrientes y vitaminas que favorece como refuerzo del sistema inmunológico, debido a que gran parte de su contenido es vitamina c fibras y minerales (Moreiras, 2009, citado en Tapia, 2020).

A nivel mundial la producción de naranjas es de 66,4 millones de toneladas aproximadamente que representa el $14 \%$, las proyecciones señalan que la producción aumentará en una tasa anual de 1,2 \% (FAO, Organización de las Naciones Unidas para la Agricultura y la Alimentación, 
2010). El cítrico es uno de los más destacados en la agricultura, y en la economía, con un estimado del 58\%, (FAO, Organización de las Naciones Unidas para la Agricultura y la Alimentación, (2013).

En Ecuador la producción total de naranja es de 149.380 TM, la superficie cultivada es de 39.860 Has. En la provincia de Bolívar la producción es de 90.092 TM con una superficie cultivada 10.630 Has., y específicamente el en cantón Caluma produce 22.482 TM con una superficie cultivada de 2.650 Has (MAGAP, Ministerio de Agricultura, Ganadería, Acuacultura y Pesca, 2013, citado en Tapia, 2020 ).

En el Cantón Caluma del subtropical de Bolívar reconocido como la capital cítrica, un árbol en tiempos atrás generaba 150 y 200 frutos, mismos que en la actualidad producen 400 y 700 frutos, esto ha generado una sobreproducción y disminución en los precios del mercado, además que, al no vender la producción total, el resto del producto se degradara, produciendo pérdidas económicas (El Telégrafo, 2019, citado en Tapia, 2020).

Reineccius, (1989 citado en Ochoa., E, 2019) el secado por atomización es uno de los métodos más empleados en muchos sectores, industriales, cerámico, químico, alimentario y farmacéutico, aplicando temperaturas de procesos adecuadas el agua se elimina en forma de vapor, hasta obtener un producto de calidad, conservando las propiedades físicos químicos de los productos, eliminar la mayor cantidad de agua y conservar el producto de los microorganismos que causan la oxidación (Geankoplis, 1998, citado en Tapia, 2020).

La simulación es la utilización de recursos computacionales con el propósito de construir modelos representativos aplicado en la industria química, física, biológica y ambiental, se basadas en operaciones unitarias, que realiza los procesos mediante diagramas de flujo, mecanizando los procesos y encontrando un punto de operación estable, que permite predecir la operación y el comportamiento real, técnica y económica del sistema (Designer, 2019, citado en Tapia, 2020).

\section{Metodología.}

La investigación es de tipo exploratoria ya que recaba información de normativas y procedimientos para la materia prima y producto final, las variables y parámetros de proceso se identifican con ensayos de laboratorio y procesos semi industriales, además del uso de la simulación computacional para la propuesta técnica y económica del diseño, basándose en los fundamentos y principios de cálculos básicos, transferencia de calor y operaciones unitarias (Ander, Egg. 1998, citado en Tapia, 2020).

Se aplicó el Diseño de Bloques Completamente Aleatorio (DBCA), se evaluaron temperaturas de proceso y concentración con un modelo factorial $1^{3}$, aplicando el análisis estadístico de varianza prueba inter-sujeto y la prueba de TUKEY. 
Figura 1. Tratamiento y diseño experimental

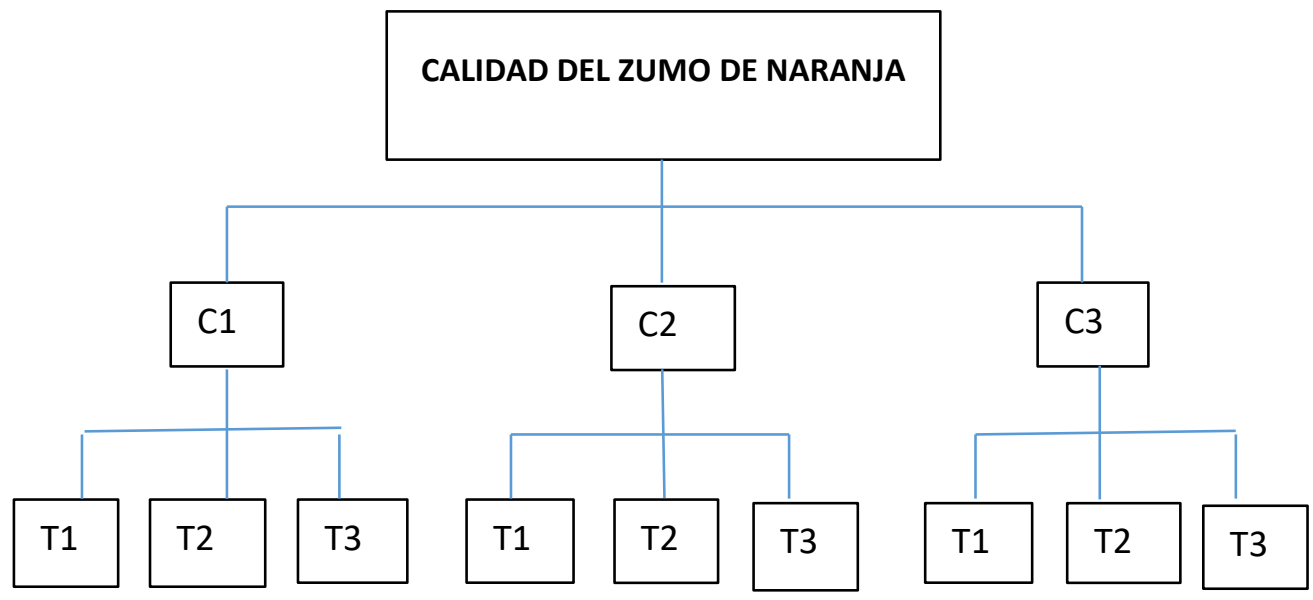

Fuente: Elaboración autores, (citado en Tapia, 2020).

Tabla 1. Variables del proceso de atomización

\begin{tabular}{lc}
\hline \multicolumn{1}{c}{ Variables } & Niveles \\
\hline Concentración & $\mathrm{C} 1 ; 5 \mathrm{C} 2 ; 7 \mathrm{C} 3 ; 9(\% \mathrm{P} / \mathrm{P})$ \\
Temperatura de proceso & $\mathrm{T} 1 ; 120, \mathrm{~T} 2 ; 140, \mathrm{~T} 3 ; 160^{\circ} \mathrm{C}$ \\
\hline
\end{tabular}

Fuente: Elaboración autores, (citado en Tapia, 2020).

\section{Descripción del proceso a nivel laboratorio.}

\section{Caracterización y selección de materia prima.}

Se caracterizó dos variedades de naranja mediante una inspección visual directa y atreves de análisis de sus parámetros fisicoquímicos fue seleccionada la naranja de variedad valencia, presentan mejores características organolépticas como: de sabor aceptable, color muy bueno, textura buena y solo presentan defectos tolerables, encontrándose libre de golpes y magulladuras en exceso, así mismo sus parámetros físicos químicos presentan un buen nivel de producción referente al diámetro, peso neto y ácido ascórbico (Tapia, 2020).

\section{Preparación de la materia prima.}

Se realizó una limpieza profunda con agua potable para remover cualquier materia extraña o contaminante que se encuentre en contactó con la fruta, posteriormente se realizó el cortado, extracción y tamización del jugo: cortando la naranja en forma ecuatorial se colocó en una mesa desinfectada cada una de las frutas, seguidamente se realizó la extracción del jugo en un extractor manual tomando en cuenta las medias higiénicas para prevenir su contaminación, simultáneamente se realizó la tamización colocando un colador sobre el recipiente recolector del jugo (Tapia, 2020). 
Según Wiseman, A. (1985 citado en Tapia., E, 2020), aplico una clarificación enzimática para regular las propiedades fisicoquímicas de la concentración del jugo de naranja (viscosidad, ${ }^{\circ} \mathrm{Brix}, \mathrm{pH}$ ) mismos que interfieren en la obtención del producto final, pues la viscosidad de las mezclas al secar por atomización afecta el tamaño de las microcápsulas y el grosor de sus paredes (Risch, 1998).

Los sólidos solubles en cantidades grandes afectan el proceso de atomización, a valores superiores a 18 grados ${ }^{\circ}$ Brix, el alimento no fluye, su manipulación y bombeo se hace difícil; afectando notablemente el proceso de secado ya que el alimento se deposita en la boquilla y la elevada temperatura del aire terminan por quemarlo (Yanza, G., 2000, citado en Tapia 2020).

Tabla 2. Propiedades físicas del jugo de naranja con tratamiento

\begin{tabular}{cccc}
\hline Parámetro & Media & Desviación estándar & Varianza \\
\hline Viscosidad (mPa.s) & 2.342 & 0.12 & 0.004 \\
Grados Brix ( ${ }^{\circ}$ Brix) & 11.675 & 0.08 & 0.005 \\
pH & 4.445 & 0.05 & 0.004 \\
\hline
\end{tabular}

Fuente: Elaboración autores, (citado en Tapia, 2020).

\section{Atomización, prueba piloto.}

En la prueba piloto se alimentó concentraciones de p/p 5\%, 7\%, y 9\%. El equipo utilizado fue un secador por atomización neumático con una boquilla de $0,002 \mathrm{~m}$, una cámara de secado de $0,58 \mathrm{~m}$ de altura, diámetro interno de $0,21 \mathrm{~m}$.

Se utilizó aire comprimido a una presión de 30 psi, a $40 \%$ de la capacidad de la bomba con un flujo de alimentación $0,25 \mathrm{~g} / \mathrm{s}$. La alimentación se dosifica en el secador por medio de una bomba de diafragma con una velocidad de $0,0113 \mathrm{~m} / \mathrm{s}$, y una potencia de 0,02 HP. Se utilizó un sistema de recuperación de ciclón separador de aire / polvo. En la prueba piloto de trabajó con temperaturas de proceso de $120{ }^{\circ} \mathrm{C}, 140{ }^{\circ} \mathrm{C}$ y $160{ }^{\circ} \mathrm{C}$ (Tapia, 2020).

\section{Descripción de la simulación.}

La simulación de la planta de producción de polvo de naranja se realizó considerando una capacidad de procesamiento de 25 TM por lote, definiendo los compuestos y las sustancias involucradas en el proceso. Se aplicó las variables y parámetros identificados en los ensayos a nivel laboratorio que permitió la producción del polvo de naranja.

El diseño del proceso de simulación se realizó en función a 5 Toneladas que corresponde al 25 $\%$ de la producción no utilizada en la comercialización de naranja del cantón Caluma, teniendo en cuenta que la producción total es de 22.482 TM (Tapia, 2020). 
Figura 2. Diagrama de flujo propuesto para producción de polvo de naranja

Producción de Jugo de Naranja en Polvo

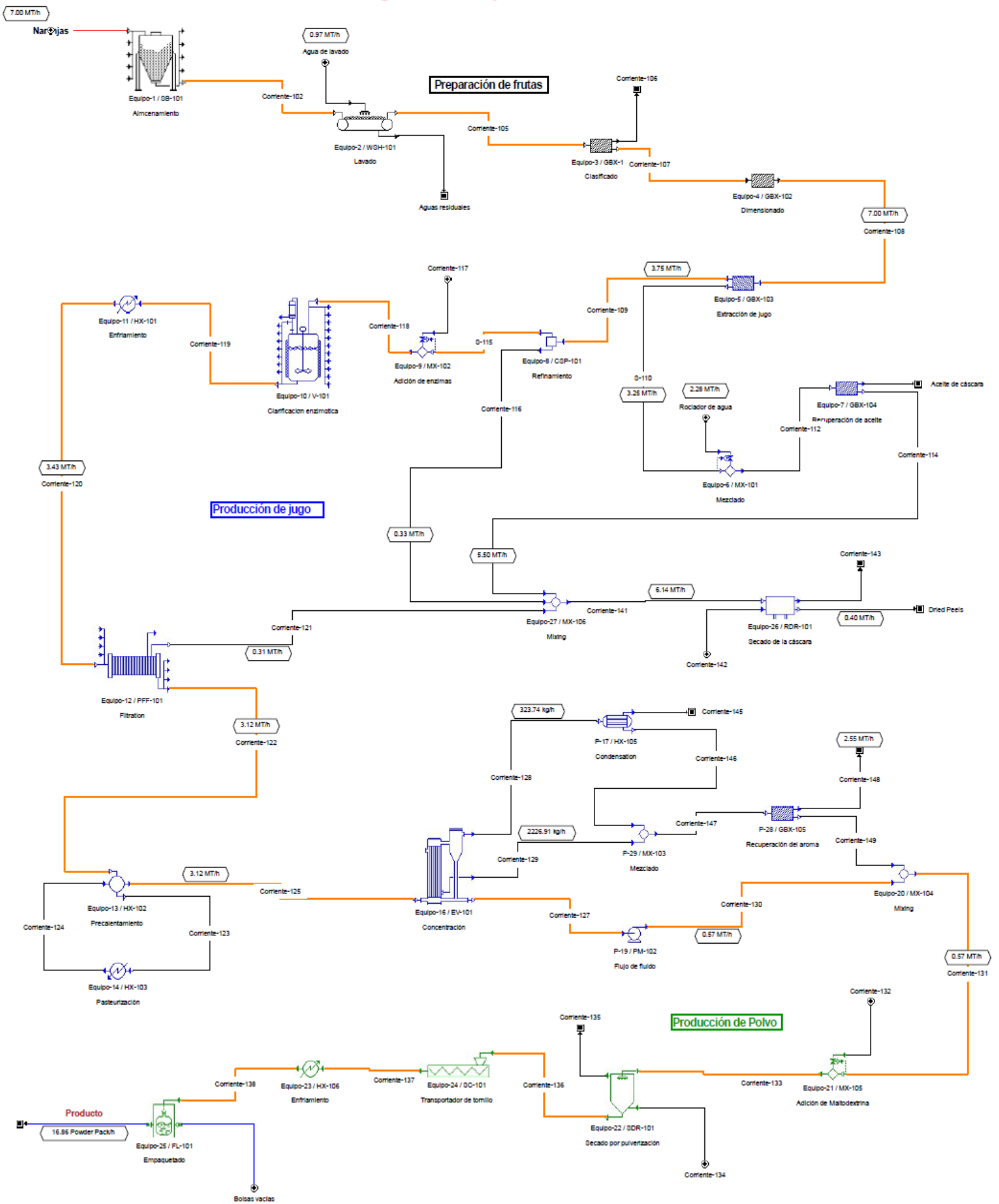

Fuente: Elaboración propia.

Según Tapia, (2020), realizó el proceso de simulación ingresando al sistema todos los componentes necesarios sustancias y compuesto que ingresan y salen del proceso, que interactúan de manera simultánea durante la producción del polvo de naranja, los parámetros del diseño de los equipos definen las condiciones de funcionamiento. 
Tabla 3. Propiedades básicas físicas de componentes (Datos del Simulador)

\begin{tabular}{lcccc}
\hline Componente & MW (g/gmol) & Tb $\left({ }^{\circ} \mathbf{C}\right)$ & Tfreez $\left({ }^{\circ} \mathbf{C}\right)$ & $\begin{array}{c}\text { DHform } \\
(\mathbf{J} / \mathbf{g m o l})\end{array}$ \\
\hline Aroma & 46,07 & 78,25 & $-114,10$ & $-276.980,00$ \\
Bentonita & 519,75 & 100,00 & 0,00 & $-285.830,00$ \\
Enzimas & 18,02 & 100,00 & 0,00 & $-285.830,00$ \\
Ácido clorhídrico & 36,46 & $-84,95$ & $-114,18$ & $-92.310,00$ \\
Maltodextrina & $3.600,00$ & 100,00 & 0,00 & $-285.830,00$ \\
Metanol & 32,00 & 64,75 & $-97,68$ & $-239.100,00$ \\
Nitrógeno & 28,02 & $-195,76$ & $-210,00$ & 0,00 \\
Oxigeno & 32,00 & $-182,84$ & $-218,79$ & 0,00 \\
Film de empaque & 18,02 & 100,00 & 0,00 & $-285.830,00$ \\
Ácido Péctico & 179,00 & 100,00 & 0,00 & $-285.830,00$ \\
Pectina & $100.000,00$ & 100,05 & $-273,15$ & 0,00 \\
Corteza & $2.100,00$ & 100,05 & $-273,15$ & 0,00 \\
Aceite de corteza & 136,23 & 100,05 & $-273,15$ & 0,00 \\
Agua de corteza & 18,02 & 100,00 & 0,00 & $-285.830,00$ \\
Agua de Proceso & 18,02 & 100,00 & 0,00 & $-285.830,00$ \\
Pulpa & 147,60 & 100,05 & $-273,15$ & 0,00 \\
Agua de Pulpa & 18,02 & 100,00 & 0,00 & $-285.830,00$ \\
Sólidos solubles & 342,30 & 477,85 & 186,00 & $-2.226 .100,00$ \\
Agua & 18,00 & 100,00 & 0,00 & $-285.830,00$ \\
\hline
\end{tabular}

Fuente: Grupo de investigación.

Según Tapia, (2020), ha definido tres secciones: preparación de la fruta, la producción de jugo y la producción de polvo, los mismos que, cuentan con operaciones unitarias propias de cada sección. Esta división en secciones permite realizar un seguimiento pormenorizado del diseño, simulación del proceso y evaluación de la prefactibilidad técnico-económica.

Al analizar las secciones del proceso, la sección de preparación de la fruta es la más simple, puesto que, en esta se encuentran las operaciones de almacenamiento, lavado, selección y dimensionamiento del fruto, las mismas que son operaciones mecánicas. Dentro de la sección de preparación del jugo, una de las operaciones más críticas es la clarificación enzimática del jugo, debido a que en la misma se la realiza en un biorreactor y la eficiencia de esta operación es muy sensible a las variables que intervienen en la misma, la cual es la temperatura, la que debe ser constante a $54{ }^{\circ} \mathrm{C}$. La otra operación crítica en la sección de preparación de jugo se encuentra en la concentración del jugo de naranja debido a que interviene la evaporación en equipos de múltiple efecto, teniendo como resultado de la simulación un total de 4 efectos. En esta sección también se presenta la recuperación del aceite y aroma, así como, secado de la cáscara de la naranja, como desecho de este proceso. En la sección de producción del polvo del jugo de naranja, se encuentra el secado por spray, operación crítica, debido a que, la temperatura define la calidad del producto final, estandarizando la temperatura final de los sólidos en $55^{\circ} \mathrm{C}$ (Tapia, 2020). 
El tipo de proceso es continuo, esto debido a que, se debe aprovechar el intercambio energético del proceso de evaporación, puesto que, constituiría una elevación de costos injustificable al realizar este proceso de producción en forma batch o semi continúa. Por otro lado, como unidad de producción se ha definido la funda de polvo de jugo de naranja con un peso total de 25.4 $\mathrm{Kg}$, esto definido, en función a la forma de comercialización internacional de este producto. Las propiedades fisicoquímicas y termodinámicas de cada uno de los componentes que intervienen en la simulación fueron definidas mediante revisión de diferentes fuentes bibliográficas (Tapia, 2020).

\section{Características de las corrientes que intervienen en la simulación.}

Las corrientes de entrada y salida que intervienen en cada uno de los procesos tienen sus características y condiciones de operación propias en función a los cambios físico y/o químicos que ocurren en cada uno de los equipos de cada proceso, tomando en consideración que, para la simulación de los procesos de clasificado, dimensionado y extracción de jugo, se utiliza una caja general que permite dividir las corrientes en función de un porcentaje de desecho o de división, donde el resultado será una o varias corrientes de salida con sus propias condiciones y características (Tapia, 2020).

\section{Balance de masa de componentes.}

La entrada y salida de componentes está perfectamente balanceada, manifestando que, en caso de los valores negativos, representan la cantidad de sustancia o compuesto que se ha generado en el proceso (Tapia, 2020).

Tabla 4. Componentes de entrada y salida del sistema

\begin{tabular}{lccc}
\hline \multicolumn{1}{c}{ Componente } & Entrada & Salida & Entrada-Salida \\
\hline Aroma & 3,484 & 3,484 & 0 \\
Bentonita & 4,266 & 4,266 & 0 \\
Enzimas & 12,798 & 12,798 & 0 \\
Ácido clorhídrico & 121 & 121 & 0 \\
Maltodextrina & 41,314 & 41,314 & 0 \\
Metanol & 0 & 2,166 & $-2,166$ \\
Nitrógeno & $33.510,629$ & $33.510,629$ & 0 \\
Oxigeno & $10.172,923$ & $10.172,923$ & 0 \\
Film de empaque & 7,550 & 7,550 & 0 \\
Ácido Péctico & 0 & 12,116 & $-12,116$ \\
Pectina & 13,063 & 0 & 13,063 \\
Corteza & 371,525 & 371,525 & 0 \\
Aceite de corteza & 41,281 & 41,281 & 0 \\
Agua de corteza & $3.715,248$ & $3.715,248$ & -0 \\
Agua de Proceso & $4.039,900$ & $4.039,900$ & 0 \\
Pulpa & 42,674 & 42,674 & 0 \\
Agua de Pulpa & 384,066 & 384,066 & 0 \\
Sólidos solubles & 470,285 & 470,285 & 0 \\
\hline
\end{tabular}




\begin{tabular}{lccc} 
Agua & $3.670,221$ & $3.669,002$ & 1,218 \\
\hline TOTAL & $56.501,345$ & $56.501,345$ & 0
\end{tabular}

Fuente: Grupo de investigación.

\section{Balance de energía de procesos.}

A continuación, se presenta el balance energético de los procesos que intervienen en el proceso de fabricación del jugo de naranja en polvo.

Tabla 5. Balance energético de procesos

\begin{tabular}{lcc}
\hline \multicolumn{1}{c}{ Proceso } & Energía $(\mathbf{k W}-\mathbf{h} / \mathbf{h})$ & Uso $(\boldsymbol{\%})$ \\
\hline Preparación de fruta & $\mathbf{2 , 1 2}$ & $\mathbf{2 , 3}$ \\
\hline Lavado & 0,72 & 0,8 \\
Clasificado & 0,70 & 0,8 \\
Dimensionado & 0,70 & 0,8 \\
\hline Producción de jugo & $\mathbf{8 1 , 5 2}$ & $\mathbf{8 7 , 5}$ \\
\hline Clarificación enzimática & 6,39 & 6,9 \\
Filtración & 0,84 & 6,9 \\
Concentración & 59,43 & 63,8 \\
Secado de cáscara & 9,38 & 10,1 \\
Extracción de jugo & 1,07 & 1,1 \\
Separación de aceite & 0,55 & 0,6 \\
Refinamiento & 3,59 & 3,9 \\
Flujo de fluido & 0,02 & 0,0 \\
Recuperación de bomba & 0,26 & 0,3 \\
\hline Producción de polvo & $\mathbf{9 , 5 5}$ & $\mathbf{1 0 , 2}$ \\
\hline Secado por pulverización & 9,13 & 9,8 \\
Transportador de tornillo & 0,14 & 0,2 \\
Empaquetado & 0,28 & 0,3 \\
\hline TOTAL & $\mathbf{9 3 , 1 8}$ & $\mathbf{1 0 0}$ \\
\hline
\end{tabular}

Fuente: Grupo de investigación.

La sección que más necesidad energética requiere es la de Producción de jugo, siendo el subproceso de Concentración el que más consume energía, esto debido a que, en el mismo se utiliza evaporadores de multiefecto. Mientras que, la sección de Preparación de fruta es la de menos consumo energético. En la sección de la Producción de polvo el subproceso de Secado por pulverización es el que mayor consumo energético tiene respectivamente (Tapia, 2020).

\section{Costo de equipos.}

Los costos de los equipos se encuentran en precios FOB (Free On Board), en función a las especificaciones propias de cada uno, relacionado con la capacidad o tamaño y el material de construcción. Recalcando que el material y el diseño de este, cumple con las normativas técnicas ASME (American Society of Mechanical Engineers), lo cual repercute directamente 
sobre el costo del equipo, observando una elevación sustancial de los mismos (Tapia, 2020).

Tabla 6. Costos de los equipos (FOB) en función de sus especificaciones

\begin{tabular}{|c|c|c|c|}
\hline Tipo & $\begin{array}{c}\text { Tamaño } \\
\text { (Capacidad) }\end{array}$ & & $\begin{array}{l}\text { Costo de } \\
\text { Compra } \\
\text { (\$/Unit) }\end{array}$ \\
\hline Secador de Spray & $1.211,42$ & $\mathrm{~L}$ & 40,000 \\
\hline Secador de Spray & 2,71 & $\mathrm{~m} 2$ & 5,000 \\
\hline Evaporador & 10,78 & $\mathrm{~m} 2$ & 100,000 \\
\hline Compartimento de sólidos & 806,39 & $\mathrm{~m} 3$ & 31,000 \\
\hline Reactor Agitado & $4.764,83$ & $\mathrm{~L}$ & 19,000 \\
\hline Divisor de Componente & $2.428,90$ & $\mathrm{~kg} / \mathrm{h}$ & 14,000 \\
\hline Condensador & 8,46 & $\mathrm{~m} 2$ & 30,000 \\
\hline $\begin{array}{l}\text { Lavadora (Flujo a } \\
\text { Granel) }\end{array}$ & $4.535,92$ & $\mathrm{~kg} / \mathrm{h}$ & 14,000 \\
\hline Caja Genérica & 907,19 & $\mathrm{~kg} / \mathrm{h}$ & 24,000 \\
\hline Intercambiador de Calor & 1,98 & $\mathrm{~m} 2$ & 5,000 \\
\hline Intercambiador de Calor & 18,75 & $\mathrm{~m} 2$ & 8,000 \\
\hline Intercambiador de Calor & 0,22 & $\mathrm{~m} 2$ & 4,000 \\
\hline Caja Genérica & $4.535,92$ & $\mathrm{~kg} / \mathrm{h}$ & 10,000 \\
\hline Mezclador & 371,19 & $\mathrm{~kg} / \mathrm{h}$ & 5,000 \\
\hline Relleno & 0,18 & entidad/min & 19,000 \\
\hline Tornillo Conveyor & 15,00 & $\mathrm{~m}$ & 2,000 \\
\hline Mezclador & $3.581,95$ & $\mathrm{~kg} / \mathrm{h}$ & 5,000 \\
\hline Caja Genérica & $3.581,95$ & $\mathrm{~kg} / \mathrm{h}$ & 10,000 \\
\hline Mezclador & $2.221,92$ & $\mathrm{~kg} / \mathrm{h}$ & 5,000 \\
\hline Mezclador & 394,20 & $\mathrm{~kg} / \mathrm{h}$ & 5,000 \\
\hline Caja Genérica & $4.535,92$ & $\mathrm{~kg} / \mathrm{h}$ & 16,000 \\
\hline Secador Rotativo & 70,25 & $\mathrm{~m} 2$ & 50,000 \\
\hline Mezclador & $3.976,33$ & $\mathrm{~kg} / \mathrm{h}$ & 5,000 \\
\hline Mezclador & $1.652,80$ & $\mathrm{~kg} / \mathrm{h}$ & 5,000 \\
\hline Caja Genérica & $1.652,80$ & $\mathrm{~kg} / \mathrm{h}$ & 19,000 \\
\hline Centrifuga Pump & 0,01 & $\mathrm{~kW}$ & 4,000 \\
\hline Intercambiador de Calor & 0,08 & $\mathrm{~m} 2$ & 7,000 \\
\hline
\end{tabular}

Fuente: Grupo de investigación.

\section{Resultados.}

Se caracterizó dos variedades de naranja analizando sus propiedades fisicoquímicas, seleccionando la variedad valencia con un $79.78 \%$ y descartando la Variedad Washington con el $61.5 \%$, se clarifico y encapsulo el fluido para su atomización. 
Tabla 7. Prueba de Tukey en relación de temperaturas

\begin{tabular}{cccc}
\hline \multicolumn{4}{c}{ Rendimiento } \\
\hline \multicolumn{4}{c}{ HSD Tukey ${ }^{\mathrm{a}, \mathrm{b}}$} \\
Temperatura & $\mathrm{N}$ & \multicolumn{2}{c}{ Subconjunto } \\
& \multicolumn{3}{c}{1} \\
Temperatura $120^{\circ} \mathrm{C}$ & 3 & 50,3667 & \\
Temperatura $160^{\circ} \mathrm{C}$ & 3 & & 64,2000 \\
Temperatura $140^{\circ} \mathrm{C}$ & 3 & & 76,7667 \\
Sig. & & 1,000 &, 134 \\
& & & \\
\hline
\end{tabular}

Fuente: Grupo de investigación.

Tabla 8. Prueba de Tukey en relación de concentración

\begin{tabular}{cccc}
\hline \multicolumn{4}{c}{ Rendimiento } \\
\hline \multicolumn{3}{c}{ HSD Tukey ${ }^{\mathrm{a}, \mathrm{b}}$} \\
Concentración & $\mathrm{N}$ & \multicolumn{2}{c}{ Subconjunto } \\
& \multicolumn{3}{c}{1} \\
Concentración 5\% & 3 & 50,300 & 2 \\
Concentración 9\% & 3 & 63,933 & \\
Concentración 7\% & 3 & & 76,420 \\
Sig. & &, 445 & 1,000 \\
\hline
\end{tabular}

Fuente: Grupo de investigación.

La aplicación de la prueba de comparaciones múltiples de TUKEY con un nivel de confianza del $95 \%$, demostraron el mejor resultado con temperatura y concentración de $140^{\circ} \mathrm{C}$ y $50 \%$ en relación p/p. con un rendimiento del producto final de $76,76 \%$ y una humedad de $0.763 \%$.

La validación del polvo de naranja que se obtuvo mediante atomización se realizó en base al rango permisible NTE INEN 2471, mezclas en polvo para preparar refrescos o bebidas instantáneas, y un producto existente y comercializado en el mercado. El resultado fue aprobado ya que los parámetros están dentro de la norma especificada. Brix; 9.6, pH; 3.61. Para coliformes Totales, coliformes fecales, E. coli presenta un valor de $0 \mathrm{UFC} / \mathrm{g}$, mientras tanto que para Mohos y Levaduras el resultado es $\leq 10 \mathrm{UPC} / \mathrm{g}$. razón por la cual el producto cumple con las expectativas deseas y es apto para su almacenamiento y consumo.

\section{Evaluación Económica Total.}

El análisis de flujo de caja ha sido desarrollado mediante la información internacional de comercialización del jugo de naranja en polvo. Además, se ha mantenido constante los ingresos de ventas y costos de operación para todos los años de recuperación de capital, solo teniendo una pequeña variación en el primer año, debido a que en este se da el mayor desembolso de capital de inversión. 
La unidad de producción utilizada es el dólar americano (\$/USD), la misma que, contiene un peso neto de producto final de $25.4 \mathrm{Kg}$ de jugo de naranja en polvo, esta cantidad de producto por USD se encuentra en función de la forma de comercialización internacional de este producto.

Tabla 9. Evaluación económica total

\begin{tabular}{ccc}
\hline Inversión Total de Capital & $2.878,000$ & (USD) \\
Costo de Operación & $4.815,000$ & (USD/año) \\
Net Costo de Operación & $4.814,762$ & (USD/año) \\
Ingresos Principales & $4.194,000$ & (USD/año) \\
Otros Ingresos & 907,446 & (USD/año) \\
Ingresos Totales & $5.102,000$ & (USD/año) \\
Costo Tasa Anual & 20,971 & (fundas/año) \\
Costo Unitario de Producción Neto & 229,59 & (USD) \\
Ingreso de Producción Unitaria & 243,27 & (USD) \\
Margen Bruto & 5,62 & $(\%)$ \\
Retorno de la Inversión & 15,28 & $(\%)$ \\
Tiempo de Retribución & 6,55 & (años) \\
TIR (Después de Impuestos) & 10,70 & $(\%)$ \\
VAN (al 7.0\% de Intereses) & 744,000 & (\$USD) \\
\hline
\end{tabular}

Fuente: Grupo de investigación.

Del análisis de los datos, mediante la experimentación y simulación del proceso de producción de jugo de naranja en polvo. Desde el punto de vista técnico, el proyecto es totalmente viable, debido a que los subprocesos son factibles de ser implementados y el producto final cumple con los requisitos necesarios para su comercialización y consumo.

El proceso de producción también es económicamente factible, sin embargo, el sistema en 15 años genera un VAN de 744.000USD y un TIR de $10 \%$ que a pesar de ser positivos podrían no ser atractivos para los inversores, considerando que se requerirá una inversión total del capital (\$2.878.000\$USD) y el costo de operación (4.814.762\$USD).

Los costos de capital fijo por cada sección del proceso de producción del jugo de naranja en polvo, es la que más capital necesita con un total de $49.45 \%$ respecto al $43.29 \%$ de la sección de preparación de fruta y el $7.27 \%$ de la producción del polvo. Mientras que, en cada sección su capital fijo tiene un costo mayor en lo que corresponde a materia prima comparado a los demás costos, con un $50.17 \%$ en la sección de preparación de frutas, $43.51 \%$ de la sección de la producción de frutas y 49.14 \% de la sección de producción de polvo. 


\section{Conclusiones:}

- La investigación se basó en un estudio a escala piloto aplicando la simulación de un proceso tecnológico, con el uso de un simulador de código abierto, en la producción del jugo de naranja en polvo, mediante atomización. Como punto de inicio del estudio, se identificó que la variedad de naranja Valencia, es la óptima para el diseño del proceso, por sus excelentes características fisicoquímicas y organolépticas de acuerdo con las normativas INEN 2844 norma para la naranja Y El CODEX STAN 245-2004, MOD.

- Para el proceso de atomización se determinó que se debe aplicar un proceso de clarificación enzimática para regular su viscosidad y ${ }^{\circ}$ Brix, considerando que el extracto presenta una elevada pegajosidad a causa de los contenidos de los sólidos solubles, mismos que sin tratamiento enzimático hacen más difícil el proceso de atomización, como agente encapsulante se utilizó la maltodextrina en relación del 50\% de p/p, para conservar sus propiedades fisicoquímicas.

- Al aplicar el Diseño de Bloques Completamente Aleatorio (DBCA), se evaluaron temperaturas de proceso y concentración tomando como variable de respuesta el rendimiento, humedad, ${ }^{\circ}$ Brix, $\mathrm{pH}$, coliformes Totales, coliformes fecales, Ecoli, Mohos y Levaduras. Mediante análisis estadístico y comparaciones según Tukey se determinó, temperatura óptima de proceso de $140{ }^{\circ} \mathrm{C}$ con una concentración del $7 \%$ p/p, con lo cual se obtiene un rendimiento del $76,76 \%$ y una humedad de $0.763 \%$.

- Los resultados del jugo de naranja en polvo como producto final están dentro del rango permisible de la norma NTE INEN 2471: 2010 mezclas en polvo para preparar refrescos o bebidas instantáneas.

- En función al $25 \%$ de la producción no utilizada en la comercialización de naranja del cantón Caluma y teniendo en cuenta que la producción total es de 22.482 TM, se realizó un diseño del proceso para 5 Toneladas anuales, como unidad de producción se ha definido la funda de polvo de jugo de naranja con un peso total de $25.4 \mathrm{Kg}$, esto definido, en función a la forma de comercialización internacional de este producto.

- Para el diseño y simulación del proceso, se ha definido tres secciones: preparación de la fruta, la producción de jugo y la producción de polvo, los mismos que, cuentan con operaciones unitarias propias de cada sección. Esta división en secciones permite realizar un seguimiento pormenorizado del diseño, simulación del proceso y evaluación de prefactibilidad técnico-económica.

\section{Referencias Bibliográficas.}

Ander, Egg. (1998). “Introducción a las Técnicas de Investigación Social”. Buenos Aires: Primera Edición, Editorial Humanistas.

Ayala., O, Solano Sosa, \& et al. (2011). Secado por atomización zumo de naranja (citrus sinensis): influencia en las variables de proceso en la perdida de vitamina c.

Designer, E. (2019). Manual de SuperPro Designer. 
El Telégrafo . (2019). La sobreproducción de naranja provoca que el precio de la fruta en finca se desplome.

FAO, Organización de las Naciones Unidas para la Agricultura y la Alimentación. (2010). "Modelo espacial del mercado mundial de cítricos elaborado en la Universidad de Florida".

FAO, Organización de las Naciones Unidas para la Agricultura y la Alimentación. (2013). Producción mundial de la naranja.

Geankoplis, J. (1998). "Procesos de Transporte y Operaciones Unitarias”. Tercera Edición.

Moreiras, O. Á. (2009 citado en Tapia, A 2020). "La alimentación española" Características nutricionales de los principales alimentos de nuestra dieta. Ministerio de Medio Ambiente y Medio Rural y Marino.

(MAGAP), Ministerio de Agricultura, Ganadería, Acuacultura y Pesca. (2013). "Informe Anual de la producción de la naranja a nivel provincial y cantonal.

Ochoa., E. (2019). Evaluación de los parámetros de secado por atomización en el contenido de vitamina $\mathrm{C}$ de microencapsulado de copoazú (Theobroma Grandiflorum). Peru.

Reineccius, G. (1989). Flavor encapsulation. Food Reviews International, 5(2), 147-176.

Risch, S. y. (1998). "Spray-dried orange oil: Effect of emulsion size on flavor retention and shelf stability". ACS Symp.

Tapia, A. (2020). Elaboración De Un Proceso Tecnológico Para La Producción De Jugo De Naranja En Polvo. Latacunga.

Wiseman, A. (1985). Manual de biotecnología del enzima. Zaragoza : Acribia.

Yanza G. (2000). "Diseño de un secador por atomización a nivel piloto para jugo concentrado de tomate de arbol". Colombia, Manizales.

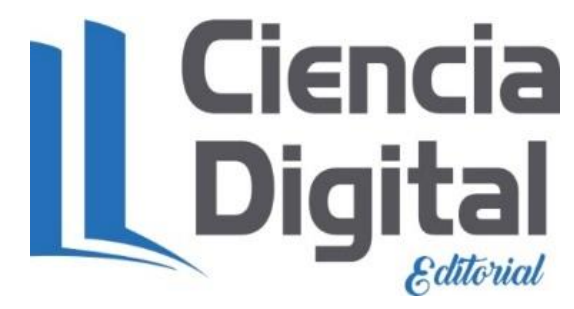




\section{PARA CITAR EL ARTÍCULO INDEXADO.}

Tapia Borja, A. I., Parada Rivera, M. M., Puente Guijarro, C. A., Manobanda Pinto, P. M., \& Guanoluisa Ataballo, G. I. (2020). Elaboración de un proceso tecnológico para la producción de jugo de naranja en polvo. Ciencia Digital, 4(3), 195-209. https://doi.org/10.33262/cienciadigital.v4i3.1323

\section{Ciencia \\ LDigital}

El artículo que se publica es de exclusiva responsabilidad de los autores y no necesariamente reflejan el pensamiento de la Revista Ciencia Digital.

El artículo queda en propiedad de la revista y, por tanto, su publicación parcial y/o total en otro medio tiene que ser autorizado por el director de la Revista Ciencia Digital.
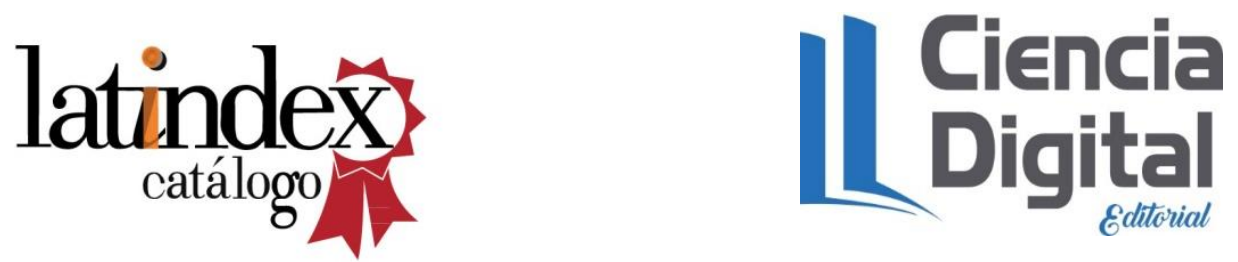